\title{
MicroRNA-21-mediated regulation of Sprouty2 protein expression enhances the cytotoxic effect of 5-fluorouracil and metformin in colon cancer cells
}

\author{
YIN-HSUN FENG ${ }^{1-3}$, CHAO-LIANG WU ${ }^{2,4}$, AI-LI SHIAU ${ }^{2,5}$, JENQ-CHANG LEE ${ }^{6}$, JAN-GOWTH CHANG ${ }^{7}$, \\ PEI-JUNG LU ${ }^{2}$, CHAO-LING TUNG ${ }^{1}$, LI-YIA FENG ${ }^{8}$, WEN-TSUNG HUANG ${ }^{9}$ and CHAO-JUNG TSAO ${ }^{9}$ \\ ${ }^{1}$ Division of Hematology and Oncology, Department of Internal Medicine, Chi-Mei Medical Center, \\ Yong Kang, Tainan 71004; ${ }^{2}$ Institute of Clinical Medicine, College of Medicine, National Cheng Kung University, \\ Tainan 70101; ${ }^{3}$ College of Medicine and Life Science, Chung Hwa University of Medical Technology, Tainan 71755; \\ Departments of ${ }^{4}$ Biochemistry and Molecular Biology, ${ }^{5}$ Microbiology and Immunology, and ${ }^{6}$ Surgery, College of \\ Medicine, National Cheng Kung University, Tainan 70101; ${ }^{7}$ Department of Laboratory Medicine, \\ Kaohsiung Medical University Hospital, Kaohsiung 80708; ${ }^{8}$ National Kaohsiung University \\ of Hospitality and Tourism, Kaohsiung 81271; ${ }^{9}$ Department of Hematology and Oncology, \\ Chi-Mei Medical Center, Liou Ying, Tainan 73657, Taiwan, R.O.C.
}

Received November 20, 2011; Accepted January 19, 2012

DOI: $10.3892 / \mathrm{ijmm} .2012 .910$

\begin{abstract}
Sprouty2 (Spry2) was identified recently as a tumor suppressor gene in cancer cells which inhibits the activation of receptor tyrosine kinases (RTKs). The present study explored the effect of Spry2 in colon cancer cells in order to assess its potential use in the treatment of colon cancer. Expression of Spry2 inhibited the growth of a colon cancer cell line, HCT116, and induced sensitization to fluorouracil (5-FU) and metformin. Spry 2 promoted apoptosis of cancer cells in association with activation of the phosphatase and tensin homolog deleted on chromosome 10 (PTEN) pathway and the blockade of Ras-Raf-Erk signaling. Treatment of Spry2-HCT116 cells with metformin resulted in a more prominent effect on the inhibition of cell migration. Inhibition of microRNA-21 (mir-21) induced upregulation of Spry2 and PTEN which underscores the importance of mir-21 in Spry2-associated tumorigenesis of the colon. These results point toward a potential strategy for colon cancer treatment worthy of further investigation.
\end{abstract}

Correspondence to: Dr Yin-Hsun Feng, Division of Hematology and Oncology, Department of Internal Medicine, Chi-Mei Medical Center, 901 Chung-Hwa Road, Yong Kang District, Tainan 71004, Taiwan, R.O.C.

E-mail: yinhsun.feng@gmail.com

Dr Chao-Jung Tsao, Department of Hematology and Oncology, Chi-Mei Medical Center, Liouying, 201, Taikang Village, Liou Ying District, Tainan 73657, Taiwan, R.O.C.

E-mail: cjt@mail.chimei.org.tw

Key words: Sprouty, microRNA-21, colon cancer, fluorouracil, metformin

\section{Introduction}

Growth factor signaling by receptor tyrosine kinases (RTKs) regulates several important cellular functions in tumor cells, such as proliferation and invasion. A negative feedback mechanism is one of the mechanisms that provide effective control via modulation of the activated RTKs pathway. Sprouty (Spry) was originally described in Drosophila as an antagonist to Breathless, the insect equivalent of fibroblast growth factor (FGF) receptor, since the loss of function of Spry led to overactive FGF signaling, hence, an excessive branching network, while forced expression of Spry blocked tracheal branching (1). Subsequent studies have demonstrated that Spry can also inhibit the Drosophila epithelial growth factor receptor (EGFR), as well as other RTKs (2-4).

Mammals have at least four isoforms of Spry protein (Spry 1-4). All Spry genes have a highly conserved C-terminal cysteine-rich domain that is important for the membrane localization of Spry family members $(5,6)$. The N-terminal domain of Spry family members is less well conserved (25-37\%) and may impart unique functions to individual family members. The N-terminal domain of Spry2 binds to c-Cbl, a ring-finger domain containing protein, and one involved in targeting the EGF receptor (7-10). Within the Spry family, Spry2 exhibits the highest level of homology to the ancestral gene. Regarding cellular functions, Spry2 owes its ability to its inhibition of Akt and Erk activation induced by some, but not all RTKs (11). Previous reports have shown that Spry2 was de-regulated in human cancer, including breast, prostate and liver cancer (12-14). Overexpression of Spry2 in osteosarcoma cancer cells inhibits proliferation and migration, implying a novel strategy in cancer treatment (15).

The first identified biochemical hallmark of tumor cells was a shift in glucose metabolism from oxidative phosphoryla- 
tion to aerobic glycolysis. In the 1920s, Warburg proposed a defect in the cellular energy of tumor cells, utilizing glycolysis instead of mitochondrial oxidative phosphorylation for glucose metabolism, even in oxygen-rich conditions (the 'Warburg effect') (16). The adenosine monophosphate (AMP)-activated protein kinase (AMPK), a master metabolic regulator, has been connected to several human tumor suppressors. It is highly conserved as a heterotrimer, and composed of an $\alpha$ catalytic kinase subunit and $\beta$ and $\gamma$ regulatory subunits. AMPK is activated under conditions of decreased intracellular ATP and increased intracellular AMP, such as nutrient deprivation or hypoxia. While activated, AMPK increases catabolic ATP-generating processes such as fatty-acid oxidation and inhibits ATP-consuming biosynthetic processes such as protein, cholesterol and fatty-acid synthesis (17). In mammalian systems, the dominant upstream kinase responsible for activating AMPK is a novel kinase named LKB1, which had been previously identified as the tumor suppressor mutated in the rare autosomal dominant Peutz-Jeghers syndrome (18). Patients with Peutz-Jeghers syndrome universally develop gastrointestinal polyps and are predisposed to several types of cancer, especially colon cancer at an early age. Genetic depletion of LKB1 in mouse embryonic fibroblasts (MEFs) results in a loss of AMPK activation following energy stresses that raise AMP (19). Metformin, a biguanide commonly used in the treatment of type 2 diabetes mellitus, recently demonstrated the ability to inhibit the breast cancer cell growth through activation of AMPK and inactivation of a mammalian target of rapamycin (mTOR) and S6 kinase (20,21). In p53 (-/-) colon cancer cells, metformin was found to increase cell apoptosis, but to activate autophagy in p53 (+/+) cells (22). Another report showed that 5-aminoimidazole-4-carboxamide ribonucleoside (AICAR), a pharmacologic activator of AMPK, enhanced apoptosis of wild-type p53 colon cancer cells co-treated with tumor necrosis factor (TNF)-related apoptosis-inducing ligand (TRAIL) (23). It is critical to explore the therapeutic strategy of metformin and its link with other treatment options in colon cancer.

\section{Materials and methods}

Cell culture. The human colon cancer cell line, HCT116, was obtained from the American Type Culture Collection and cultured in Dulbecco's modified Eagle's medium/F12 with $10 \%$ fetal bovine serum.

Reagents. Fluorouracil (5-FU) and methylthiazolydiphenyltetrazolium bromide (MTT) powder were purchased from Sigma-Aldrich (USA). Metformin was obtained from Alexis (Germany) and was dissolved in water. Anti-phosphorylatedAMPK, anti-phosphorylated Akt, and anti-total Akt were from Cell Signaling (USA). Anti-PTEN was obtained from Santa Cruz Biotechnology, Inc., and anti- $\alpha$-tubulin was purchased from Sigma-Aldrich. Horseradish peroxidase-conjugated antirabbit IgG and anti-mouse IgG were from Pierce (USA). the Annexin $\mathrm{V}$ kit and the enhanced chemiluminescencent (ECL) were from Roche Diagnostic (USA). Anti-poly (ADP-ribose) polymerase (PARP) was from BD Pharmingen (USA) and anti-BCL2 was from Transduction Laboratories (USA). AntiSpry2 was obtained from Upstate Biotechnology (USA).
Constructs and transfection. Human Spry2 full-length cDNA was inserted in a pEGFP-N3 (Spry2-GFP) vector (15) as a kind gift from Vande Woude, Van Andel Research Institute. HCT116 cells were transfected with Spry2-GFP expression plasmid (Spry2-HCT116) and Lipofectamine (Invitrogen, USA), as described in the manufacturer's standard procedure. HCT116 cells transfected with pEGFP-N3 empty vector were regarded as vector-HCT116 cells.

Cytotoxicity assay. The cytotoxity of cells was assessed by the MTT assay. In brief, HCT116 colon cancer cells were transfected with Spry2-GFP plasmid or vector only plasmid, using the Lipofectamine method. Forty-eight hours after transfection, Spry2-HCT116 and vector-HCT116 were cultured in a 96-well plate (Corning, Inc., USA) at a density of 4,000 cells/ well in DMEM/F12 with $10 \%$ FBS (controlled medium). After $24 \mathrm{~h}$ incubation, $25 \mu \mathrm{M}$ of $5-\mathrm{FU}$ or $20 \mathrm{mM}$ of metformin was added to DMEM/F12 with $10 \%$ FBS, in a total volume of $200 \mu \mathrm{l} / \mathrm{well}$, to replace the original DMEM/F12. After $96 \mathrm{~h}, 20 \mu \mathrm{l}$ of $5 \mathrm{mg} / \mathrm{ml}$ MTT solution was added to each well. Plates were incubated in the dark for $2 \mathrm{~h}$ and then the crystal was eluted with $100 \mu \mathrm{l}$ DMSO after removal of the culture medium and the MTT solution. The absorbance was measured at $570 \mathrm{~nm}$ using a microtiter plate reader (MRX Revelation, Dynex, USA).

Annexin V staining, propidium iodide staining and flow cytometry. The HCT116 cells were transfected with Spry2-GFP or vector-only plasmid, using Lipofectamine. Approximately $1 \times 10^{6}$ Spry2-HCT116 or vector-HCT116 cells were plated in each well in 6-well plates and incubated for $16 \mathrm{~h}$. Then the cells were treated with either $20 \mathrm{mM}$ of metformin, or $25 \mu \mathrm{M}$ of 5-FU for $48 \mathrm{~h}$ with DMEM/F12 and 10\% FBS. The cell culture medium containing floating cells was collected. Attached cells were rinsed with $\mathrm{Ca}^{2+}$ and $\mathrm{Mg}^{2+}$-free PBS and harvested after incubation with $2 \mathrm{mM}$ EDTA in PBS for $10 \mathrm{~min}$. Floating and attached cells were pooled and rinsed twice with PBS. Cells were resuspended in $1 \mathrm{X}$ binding buffer (Axxora Platform, USA) and incubated with FITC-conjugated Annexin V for $15 \mathrm{~min}$ at room temperature in the dark, using the FITC-Annexin V apoptosis detection kit (Roche Diagnostic). Cells were washed twice with $1 \mathrm{X}$ binding buffer. The samples were immediately analyzed on FACSCalibur flow cytometer (Becton-Dickinson Immunocytometry System, USA) using the CellQuest Pro Software and the WinMDI 2.9 software. Propidium iodide was added right before the analysis of these samples.

Protein extraction and western blot analysis. HCT116 cells were transfected with Spry2-GFP plasmid or vector-only plasmid (control), using Lipofectamine. Twenty-four hours after transfection, cells were treated with either controlled medium (DMEM/F12 with 10\% FBS), controlled medium with $25 \mu \mathrm{M}$ of $5-\mathrm{FU}$, or controlled medium with $5 \mathrm{mM}$ of metformin, for $48 \mathrm{~h}$. Cells were washed twice with PBS and lysed in $300 \mu \mathrm{l}$ lysis buffer $(20 \mathrm{mM}$ Tris- $\mathrm{HCl}, \mathrm{pH} 8.0,100 \mathrm{mM}$ $\mathrm{NaCl}, 1 \mathrm{mM}$ EDTA, $0.5 \%$ nonidet P40, 0.5\% Triton $\mathrm{X}-100$, $1 \mathrm{mM}$ phenylmethylsulfonyl fluoride, $1 \mathrm{mM} \mathrm{NaF}, 1 \mathrm{mM}$ sodium orthoyanadate, $1 \mathrm{mg}$ each of aprotinin, leupeptin, and pepstatin/ml) and were processed as described previously (24). Separation of protein was performed on $10 \%$ polyacrylamide 
A

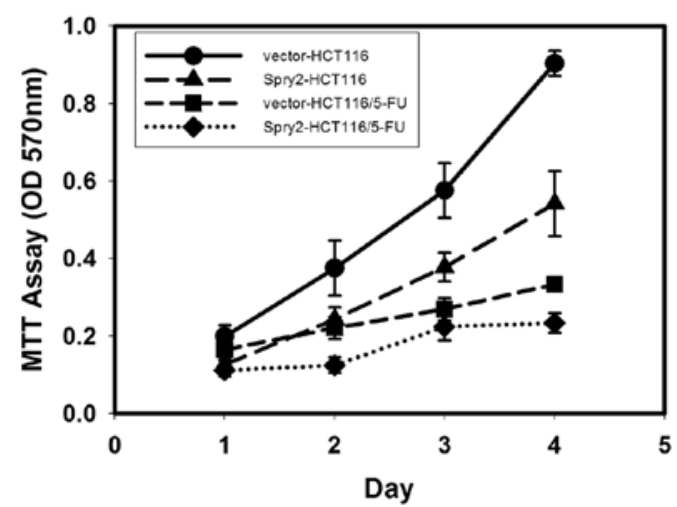

B

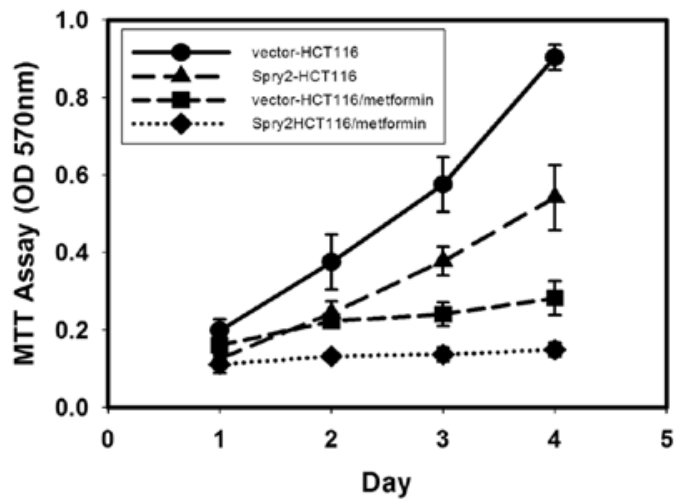

Figure 1. Spry2 expression inhibits the proliferation of colon cancer cells, HCT116 and enhances the cytotoxic effect of 5-FU and metformin. The proliferation was measured by the MTT assay on four consecutive days and in triplicate. (A) HCT116 transfected cells with Spry2 showed a lower proliferation rate compared with vector-HCT116 in a controlled medium. 5-FU in the concentration of $25 \mu \mathrm{M}$ suppressed HCT116 cell growth, and Spry2-HCT116 treated with 5-FU demonstrated the slowest growth rate. (B) Metformin, $20 \mathrm{mM}$, suppressed the growth of HCT116 cells; the inhibitory effect was more significant when Spry2-HCT116 was treated with metformin.

gels with 5\% polyacrylamide stacking gel. After electrophoretic transfer (Amersham Pharmacia Biotech, USA) of protein from the SDS polyacrylamide gel to polyvinylidene difluoride membranes (Millipore, USA), the membranes were blocked with buffer containing $20 \mathrm{mM}$ Tris- $\mathrm{HCl}, \mathrm{pH} 7.5,150 \mathrm{mM}$ $\mathrm{NaCl}, 0.05 \%$ Tween-20, 5\% Blotto (Bio-Rad, USA) at $4{ }^{\circ} \mathrm{C}$ overnight and probed with antibodies of interest. Horseradish peroxidase-conjugated anti-rabbit IgG and anti-mouse IgG were used as secondary antibodies. The position of the protein was visualized using the ECL reagent.

Cell migration/wound-healing assay. For the wound-healing assay, $48 \mathrm{~h}$ after transfection with Spry2-GFP or vector-only plasmid using Lipofectamine, transfected cells were plated separately overnight to achieve a confluent cell layer in a 12-well plate. Then, the medium was replaced with DMEM/F12 and $10 \%$ FBS (controlled medium) with or without metformin in the concentration of $20 \mathrm{mM}$. After making a scratch on the cell layer with a needle tip, wound-healing was visualized by monitoring the migrating cells in the gap during a $24-\mathrm{h}$ postscratch period.

Inhibition of microRNA-21 expression. The endogenous microRNA-21 (mir-21) was suppressed by transfection of the mir-21 inhibitor (Qiagen, Germany) in colon cancer cells According to the manufacturer's protocol, $10.3 \mu 1 \mathrm{mir}-21$ inhibitor ( $20 \mu \mathrm{M}$ stock) was diluted in $100 \mu \mathrm{l}$ culture medium without serum. Twenty microliters HiPerFect Transfection Reagent (Qiagen) was added to the diluted mir-21 inhibitor. The samples were incubated for $10 \mathrm{~min}$ at room temperature $\left(15-25^{\circ} \mathrm{C}\right)$ to allow the formation of transfection complexes. The complexes were added drop-wise onto the cells. The cells were incubated with the transfection complexes under their normal growth conditions.

Statistical analysis. Significance was assessed by a two-tailed Student's t-test. All data represent the average of a least three independent experiments.

\section{Results}

Spry2 enhances the cytotoxic effect of metformin and 5-FU in colon cancer cells. 5-FU is known to have an inhibitory effect on the proliferation of colon cancer and thus has become part of the chemotherapy regimen widely used in the treatment of patients with colon cancer. To explore the antiproliferative effect of Spry2, HCT116 colon cancer cells transfected with Spry 2 were treated with 5-FU in the concentration of $25 \mu \mathrm{M}$. The growth of Spry2-HCT116 and vector-HCT116 cells was measured by the MTT assay on 4 consecutive days. HCT116 cells with ectopic expression of Spry2 showed a lower proliferation rate and 5-FU demonstrated a better cytotoxic effect on Spry2-HCT116 cells (Fig. 1A). Metformin and other AMPK activators have been shown to suppress the growth of cancer cells, such as breast cancer and colon cancer. Herein, we demonstrated that metformin at the concentration of $20 \mathrm{mM}$ can inhibit the proliferation of HCT116 colon cancer cells. Overexpression of Spry2 in HCT116 cells led to a greater cytotoxic effect of metformin (Fig. 1B).

Spry2 enhances the cytotoxic effect of 5-FU or metformin through suppression of Akt and Erk activation followed by induction of cell apoptosis. We utilized flow cytometry and western blot analysis to determine the regulatory mechanism of Spry2 in promoting cancer cell death. After treatment with 5-FU, metformin, or a controlled medium for $48 \mathrm{~h}$, the Annexin V stain of Spry2- and vector-HCT116 cells was measured by flow cytometry (Fig. 2A). Overexpression of Spry2 in HCT116 cells induced cell apoptosis, compared with vector-HCT116 cells in a controlled medium (Spry2-HCT116, $4.233 \pm 0.666 \%$; vector-HCT116, 1.76 $\pm 0.122 \%, \mathrm{P}<0.01)$. Spry2-HCT116 cells treated with 5-FU showed a higher percentage of Annexin $\mathrm{V}$ positive cells, compared with vector-only cells treated with 5-FU (Spry2-HCT116+5-FU, $8.913 \pm 0.335 \%$; vector-HCT116+5-FU, $4.763 \pm 0.292 \%$, $\mathrm{P}<0.01)$. On the other hand, vector-HCT116 cells treated with metformin, $20 \mathrm{mM}$, did not induce more apoptosis than 
A
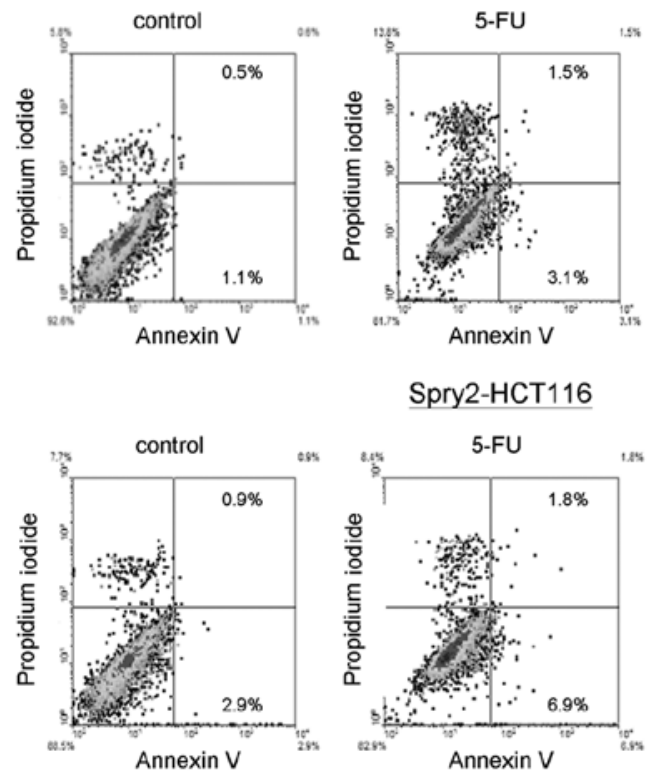

Spry2-HCT116

vector-HCT116
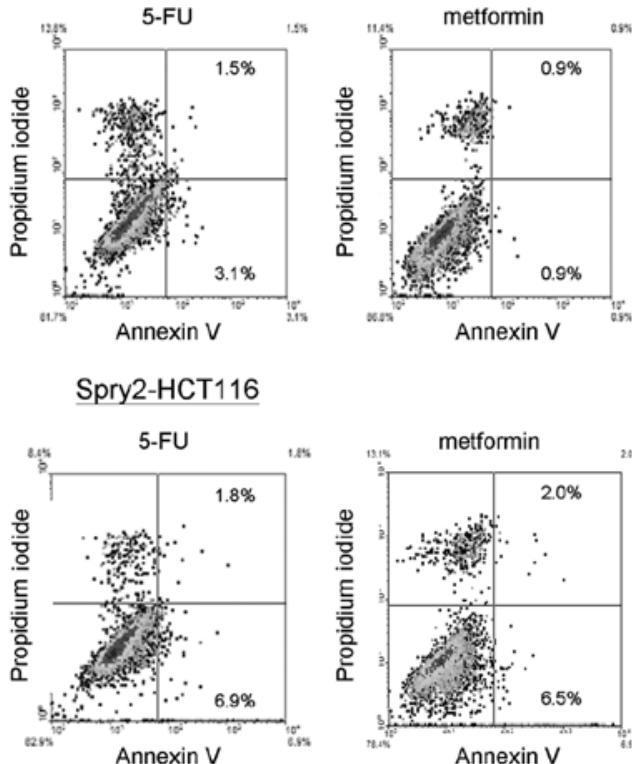

B

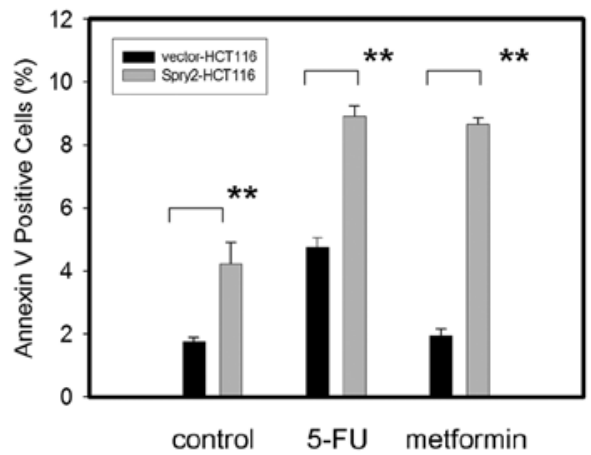

Figure 2. Spry2 expression enhances the inhibitory effect of 5-FU and metformin through cell apoptosis in HCT116 cells. (A) Positive Annexin V cells were measured by flow cytometry. In vector-HCT116 cells, treatment with 5-FU, but not metformin, could induce cells to apoptosis by increasing the percentage of Annexin V-positive cells. Increasing the expression of Spry2 in HCT116 cells promoted apoptosis under all three conditions. (B) The bar graph demonstrates that vector-HCT116 cells treated with 5-FU increased the percentage of Annexin V positive cells from 1.6 to $4.6 \%$, but the same effect was not shown in vectorHCT116 treated with metformin. HCT116 cells transfected with Spry2 promoted apoptosis from 1.6 to 3.8\%. Spry2-HCT116 cells expressing Annexin V increased from 4.6 to $8.4 \%$ in 5-FU-containing medium and from 1.8 to $8.5 \%$ in metformin-containing medium. Results represent the mean \pm SD of three experiments performed in triplicate $\left({ }^{* *} \mathrm{P}<0.01\right)$.

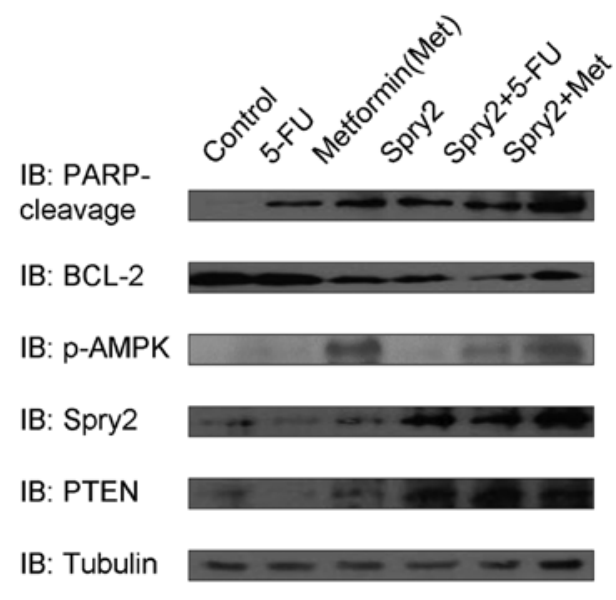

Figure 3. Spry2 protein increases the expression of PTEN and enhances cell apoptosis induced by 5 -FU or metformin in HCT116 cells. PARP cleavage indicated activation of the apoptotic pathway and BCL-2 protein represented the index of anti-apoptosis. 5-FU can induce HCT116 cell apoptosis via activation of PARP. The phosphorylation of AMPK was activated by meformin, which led to HCT116 cell apoptosis. Ectopic expression of Spry2 protein enhanced HCT116 cell apoptosis by increasing the expression of PTEN protein. The addition of metformin in Spry2-HCT116 cells resulted in the activation of AMPK and enhancement of the AKT pathway suppressor, PTEN.

vector-HCT116 cells in a controlled medium. However, after being transfected with Spry2 and treated with $20 \mathrm{mM}$ of metformin, Spry2-HCT116 cells showed significantly more Annexin V positive cells compared with vector-HCT116 cells in metformin (Spry2-HCT116+metformin, 8.663 $\pm 0.21 \%$; vector-HCT116+metformin, $1.937 \pm 0.228 \% \mathrm{P}<0.01$ ) (Fig. 2B).
Using western blot analysis to demonstrate protein regulation, metformin induced PARP cleavage and decreased BCL-2 expression, leading to cell apoptosis, which corresponds to the net result of activation of AMPK and inactivation of Akt. Spry2 protein has been known to inhibit cell growth by inhibiting activation of the Ras/Raf/Erk pathway as a negative regulator. This study has demonstrated that Spry2 can also suppress the activation of the Akt pathway by inducing expression of PTEN, an important tumor suppressor (Fig. 3). These results confirmed that Spry2 can enhance the originally suppressive effect of either 5-FU or metformin in colon cancer cells.

Spry2 enhances the migration inhibition of metformin in colon cancer cells. It is known that Spry2 can inhibit tumor proliferation and migration, but there is limited information about whether treatment with metformin in Spry2 overexpressed cancer cells generates better inhibition on cell migration, so we used a wound-healing assay to test this hypothesis. The migration distance was measured by a timelapse microscope camera within $24 \mathrm{~h}$. The results showed that expression of Spry2 can inhibit migration of HCT116 cells in a controlled medium (Spry2-HCT116, 15.462 $\pm 1.469 \mu \mathrm{m} / \mathrm{h}$; vector-HCT116, 21.691 $\pm 2.475 \mu \mathrm{m} / \mathrm{h}, \mathrm{P}<0.05)$. The suppressive effect of Spry2 in cell migration of HCT116 was also demonstrated in a controlled medium with $20 \mathrm{mM}$ of metformin (Spry2-HCT116+metformin, $11.170 \pm 1.587 \mu \mathrm{m} / \mathrm{h}$; vector-HCT116+metformin, $15.811 \pm 1.998 \mu \mathrm{m} / \mathrm{h}, \mathrm{P}<0.05$ ) (Fig. 4). This implies that both strategies can work together to achieve better results in the control of tumor growth and migration. 
A
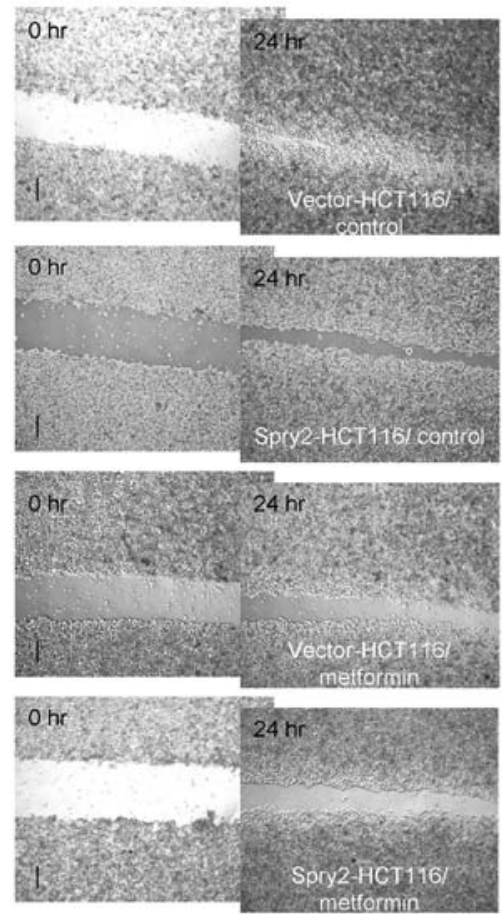

B

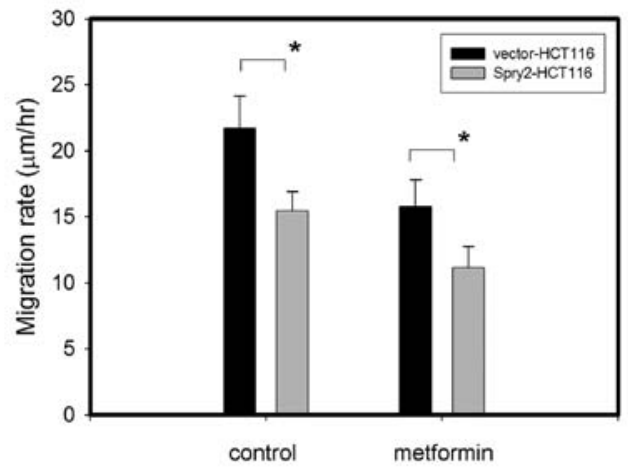

Figure 4. Spry2 enhances the inhibitory effect of metformin on the migration of colon cancer HCT116 cells. The migration of the experimental cells was measured by time-lapse microscopy within $24 \mathrm{~h}$ in triplicate. Each black bar represents $100 \mu \mathrm{m}$. (A) The migration distance decreased in Spry2-HCT116 cells in controlled medium (control) with metformin or without metformin, compared with vector-HCT116 cells. (B) The bar graph shows that the vector-HCT116 cells in controlled medium (control) migrated at $21.691 \mu \mathrm{m} / \mathrm{h}$, which was more than the Spry2-HCT116 cells in controlled medium, at $15.462 \mu \mathrm{m} / \mathrm{h}$. After treatment with metformin, the migration rate of vector-HCT116 cells decreased to $15.811 \mu \mathrm{m} / \mathrm{h}$, compared with vector-HCT116 cells in a controlled medium. Spry2-HCT116 in metformin demonstrated the slowest migration rate, at $11.170 \mu \mathrm{m} / \mathrm{h}$. The result disclosed that treatment with metformin or expression of Spry2 in HCT116 cells can inhibit the migration ability $\left({ }^{*} \mathrm{P}<0.05\right)$.

A

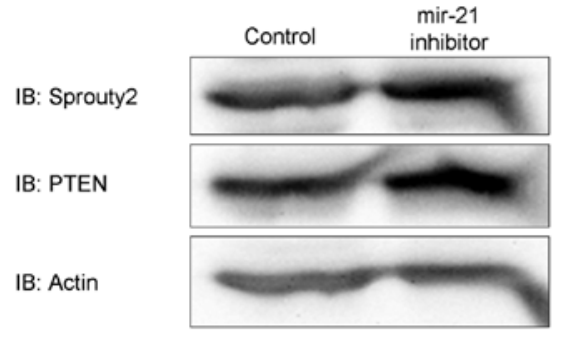

B

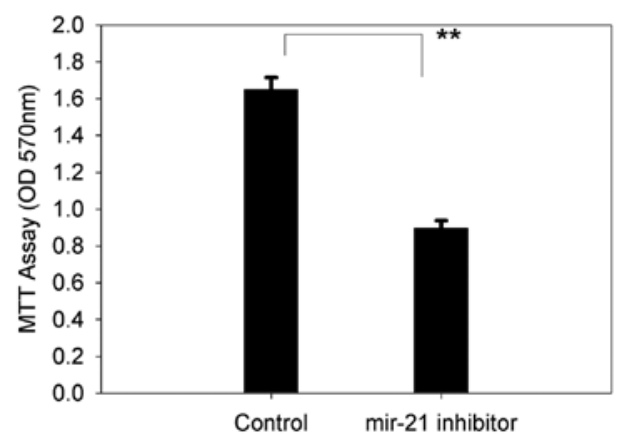

Figure 5. Suppression of mir-21 enhanced the expression of Spry2 and PTEN. (A) In HCT116 colon cancer cells, the protein expression of Spry2 and PTEN increased after mir-21 was suppressed by the mir-21 inhibitor. (B) The proliferation of HCT116 cells decreased after suppression of mir-21 by the mir-21 inhibitor $\left(^{* * *} \mathrm{P}<0.01\right)$.

Suppression of mir-21 leads to increased expression of Spry2 and PTEN in accordance with a reduced proliferation of colon cancer cells. Our previous study had shown that expression of Spry2 is reversely correlated with expression of mir-21 in tumor samples of colon cancer patients (25). It is still unclear that mir-21 has any influence on the Spry2 induced proliferation of colon cancer cells. In colon cancer cells, HCT116, mir-21 knockdown was associated with increasing expression of Spry2 and PTEN by western blot analysis (Fig. 5A). The proliferation rate of colon cancer cells was reduced after the inhibition of mir-21 (Fig. 5B). These results correlated with the oncogenic effect of mir-21 in colon cancer as previously reported (26-28).

\section{Discussion}

There is emerging evidence indicating that Spry2 protein is downregulated in cancer cells and that the ectopic expression of Spry2 can inhibit cancer cell growth $(12,13,15)$. Spry2 tumor suppression is possibly induced through inactivation of the Erk and Akt pathways. Resistance to primary therapy with drugs or medication is a problem requiring urgent attention in cancer treatment. More and more studies are focusing on conquering this tough problem through multi-pathway inhibition. Spry2 is a possible candidate for this strategy since it is a novel target which can suppress many RTKs, including fibroblast 


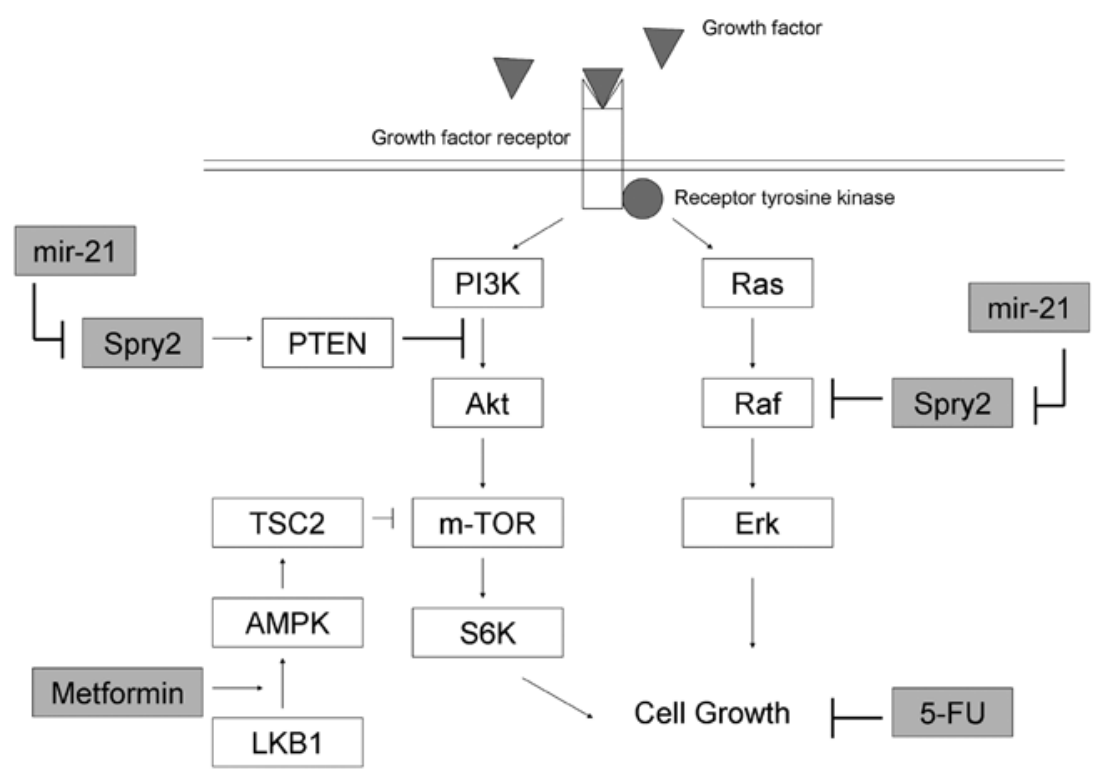

Figure 6. A schematic representation of the inhibitory effects of Spry2 and metformin on the growth factor receptor pathway. Spry2 has been shown to inhibit Akt activation by enhancement of PTEN expression and suppression of the Ras/Raf/Erk pathway. mir-21 suppresses the expression of Spry2 as demonstrated in our study. Metformin inhibits the activation of m-TOR by increasing the phosphorylation of AMPK and the activation of TSC2. The combination of different therapeutic strategies, including expression of Spry2, metformin and 5-FU, are expected to have a promising effect on colon cancer treatment.

growth factor receptor and vascular epithelial growth factor receptor, and it also directly suppresses downstream effectors of RTKs, such as Ras, Raf and Erk. Spry2 protein may act as a competitive inhibitor of Erk signaling through at least two potential mechanisms. The $\mathrm{COOH}$ terminus of Spry 2 can bind to RAF1, inhibiting kinase activity (29). Furthermore, Spry2 protein uncouples receptor tyrosine kinase signaling from the activation of Ras, possibly by sequestration of GRB2, an adaptor protein required for Ras activation $(30,31)$. For the Akt pathway, PTEN is activated by Spry2, which leads to inactivation of Akt signaling. The downregulation of Spry 2 in cancer cells may confer a proliferative advantage to tumor cells by allowing unchecked activation of the Ras/Erk pathway, which would normally be controlled by relatively higher expression levels of Spry2 in normal tissue. Several mechanisms may account for the reduced Spry 2 expression in cancer cells and the association with carcinogenesis. Accordingly, in cells lines derived from non-small cell lung carcinoma, metastatic lung cancer cells tend to have a lower expression of Spry2. In addition, an inverse correlation between Spry expression and tumor grade was observed in prostate cancer (13). Actually, Spry2 has a potential antitumor property, but so far no data have shown that Spry2 can enhance other cancer treatment strategies. Our results showed that Spry2 was able to promote apoptosis in colon cancer cells and enhance the cytotoxic effect of the chemotherapeutic agent, 5-FU. 5-FU is the time-tested agent for systemic chemotherapy for colon cancer; however, the objective response rate to $5-\mathrm{FU}$ is $15-20 \%$. The therapeutic effect of 5-FU can be enhanced by combining 5-FU and other agents, such as leucovorin, oxaliplatin and irinotecan. Activation of growth factor receptor in colon cancer has been given greater attention, and specific treatment for epidermal growth factor receptor and vascular growth factor has been deployed to manage patients with metastatic colon cancer. The Spry2 mediated enhancement of the cytotoxic benefit of the
5-FU regimen implies that Spry2 may be a surrogate marker for prediction of tumor response and may play an important role in resistance to chemotherapy.

Spry2 also has the ability to generate a better effect with metformin, an AMPK activator. AMPK is known as a master negative regulator in the protein synthesis of various cells, and it has been considered as a novel therapeutic target for the treatment of cancer and other metabolic diseases (32). Recent studies on the effects of metformin on the cell signaling network have extended from the context of diabetes research to the regulation of cancer cells. The cell proliferative effects of activated AMPK via the Akt/mammalian target of rapamycin (m-TOR) or phosphatidylinositol 3-kinase (PI3K) signaling were shown in endothelial cells. In breast cancer cells, the inactivation of LKB1 by reactive lipid species was shown to inhibit the phosphorylation of AMPK, as well as the downstream Tuberous sclerosis protein (TSC)-mTOR-S6K cascade (33). Here we demonstrated metformin alone can inhibit the proliferation and migration of colon cancer cells. Cell apoptosis, demonstrated by Annexin V assay, was not increased after metformin treatment, which can be partially explained by autophagocytosis. However, the combination of Spry2-active cancer cells and metformin treatment resulted in the additional benefit of cell killing. These data support the current concept of inactivation of oncogenic pathways and suggest a the central role of Spry2 in cancer treatment (Fig. 6).

\section{Acknowledgements}

We appreciate the grant support from the National Cheng Kung University Hospital, Taiwan (research fund 2004 no. 21), the National Science Council, Taiwan (095JFA09007), and the Chi-Mei Medical Center, Taiwan (research fund CLFHR9914 and CMFHR10006). 


\section{References}

1. Hacohen N, Kramer S, Sutherland D, Hiromi Y and Krasnow MA Sprouty encodes a novel antagonist of FGF signaling that patterns apical branching of the Drosophila airways. Cell 92: 253-263, 1998.

2. Casci T, Vinos J and Freeman M: Sprouty, an intracellular inhibitor of Ras signaling. Cell 96: 655-665, 1999.

3. Kramer S, Okabe M, Hacohen N, Krasnow MA and Hiromi Y: Sprouty: a common antagonist of FGF and EGF signaling pathways in Drosophila. Development 126: 2515-2525, 1999.

4. Reich A, Sapir A and Shilo B: Sprouty is a general inhibitor of receptor tyrosine kinase signaling. Development 126: 4139-4147, 1999.

5. Lim J, Wong ES, Ong SH, Yusoff P, Low BC and Guy GR Sprouty proteins are targeted to membrane ruffles upon growth factor receptor tyrosine kinase activation. Identification of a novel translocation domain. J Biol Chem 275: 32837-32845, 2000.

6. Lim J, Yusoff P, Wong ES, et al: The cysteine-rich sprouty translocation domain targets mitogen-activated protein kinase inhibitory proteins to phosphatidylinositol 4,5-bisphosphate in plasma membranes. Mol Cell Biol 22: 7953-7966, 2002.

7. Egan JE, Hall AB, Yatsula BA and Bar-Sagi D: The bimodal regulation of epidermal growth factor signaling by human Sprouty proteins. Proc Natl Acad Sci USA 99: 6041-6046, 2002.

8. Fong CW, Leong HF, Wong ES, Lim J, Yusoff P and Guy GR: Tyrosine phosphorylation of Sprouty2 enhances its interaction with $\mathrm{c}-\mathrm{Cbl}$ and is crucial for its function. J Biol Chem 278: 33456-33464, 2003.

9. Hall AB, Jura N, DaSilva J, Jang YJ, Gong D and Bar-Sagi D: hSpry2 is targeted to the ubiquitin-dependent proteasome pathway by c-Cbl. Curr Biol 13: 308-314, 2003.

10. Rubin C, Litvak V, Medvedovsky H, Zwang Y, Lev S and Yarden Y: Sprouty fine-tunes EGF signaling through interlinked positive and negative feedback loops. Curr Biol 13: 297-307, 2003

11. Edwin F, Singh R, Endersby R, Baker SJ and Patel TB: The tumor suppressor PTEN is necessary for human Sprouty 2-mediated inhibition of cell proliferation. J Biol Chem 281: 4816-4822, 2006.

12. Fong CW, Chua MS, McKie AB, et al: Sprouty 2, an inhibitor of mitogen-activated protein kinase signaling, is downregulated in hepatocellular carcinoma. Cancer Res 66: 2048-2058, 2006.

13. Fritzsche S, Kenzelmann M, Hoffmann MJ, et al: Concomitant downregulation of SPRY1 and SPRY2 in prostate carcinoma. Endocr Relat Cancer 13: 839-849, 2006.

14. Lo TL, Yusoff P, Fong CW, et al: The ras/mitogen-activated protein kinase pathway inhibitor and likely tumor suppressor proteins, sprouty 1 and sprouty 2 are deregulated in breast cancer. Cancer Res 64: 6127-6136, 2004.

15. Lee CC,Putnam AJ, Miranti CK, et al: Overexpression of sprouty 2 inhibits HGF/SF-mediated cell growth, invasion, migration, and cytokinesis. Oncogene 23: 5193-5202, 2004.

16. Warburg O: On the origin of cancer cells. Science 123: 309-314, 1956.
17. Shaw RJ: Glucose metabolism and cancer. Curr Opin Cell Biol 18: 598-608, 2006.

18. Alessi DR, Sakamoto K and Bayascas JR: LKB1-dependent signaling pathways. Annu Rev Biochem 75: 137-163, 2006.

19. Shaw RJ, Kosmatka M, Bardeesy N, et al: The tumor suppressor LKB1 kinase directly activates AMP-activated kinase and regulates apoptosis in response to energy stress. Proc Natl Acad Sci USA 101: 3329-3335, 2004.

20. Dowling RJ,Zakikhani M, Fantus IG, Pollak M and Sonenberg N: Metformin inhibits mammalian target of rapamycin dependent translation initiation in breast cancer cells. Cancer Res 67: 10804-10812, 2007.

21. Zakikhani M, Dowling R, Fantus IG, Sonenberg N and Pollak M: Metformin is an AMP kinase-dependent growth inhibitor for breast cancer cells. Cancer Res 66: 10269-10273, 2006.

22. Buzzai M, Jones RG, Amaravadi RK, et al: Systemic treatment with the antidiabetic drug metformin selectively impairs p53-deficient tumor cell growth. Cancer Res 67: 6745-6752, 2007.

23. Su RY, Chao Y, Chen TY, Huang DY and Lin WW: 5-Aminoimidazole-4-carboxamide riboside sensitizes TRAILand $\mathrm{TNF} \alpha$-induced cytotoxicity in colon cancer cells through AMP-activated protein kinase signaling. Mol Cancer Ther 6: 1562-1571, 2007.

24. Lee MH, Reynisdottir I and Massague J: Cloning of p57KIP2, a cyclin-dependent kinase inhibitor with unique domain structure and tissue distribution. Genes Dev 9: 639-649, 1995.

25. Feng YH, Wu CL, Tsao CJ, et al: Deregulated expression of sprouty2 and microRNA-21 in human colon cancer: Correlation with the clinical stage of the disease. Cancer Biol Ther 11: 111-121, 2011.

26. Schetter AJ, Leung SY, Sohn JJ, et al: MicroRNA expression profiles associated with prognosis and therapeutic outcome in colon adenocarcinoma. JAMA 299: 425-436, 2008.

27. Chang KH, Miller N, Kheirelseid EA, et al: MicroRNA-21 and PDCD4 expression in colorectal cancer. Eur J Surg Oncol 37: 597-603, 2011.

28. Nielsen BS, Jorgensen S, Fog JU, et al: High levels of microRNA-21 in the stroma of colorectal cancers predict short disease-free survival in stage II colon cancer patients. Clin Exp Metastasis 28: 27-38, 2011.

29. Sasaki A, Taketomi T, Kato R, et al: Mammalian Sprouty4 suppresses Ras-independent ERK activation by binding to Raf1. Nat Cell Biol 5: 427-432, 2003.

30. Hanafusa H, Torii S, Yasunaga T and Nishida E: Sprouty1 and Sprouty 2 provide a control mechanism for the Ras/MAPK signalling pathway. Nat Cell Biol 4: 850-858, 2002.

31. Gross I, Bassit B, Benezra M and Licht JD: Mammalian sprouty proteins inhibit cell growth and differentiation by preventing ras activation. J Biol Chem 276: 46460-46468, 2001.

32. Kim HJ and Bar-Sagi D: Modulation of signalling by Sprouty: a developing story. Nat Rev Mol Cell Biol 5: 441-450, 2004.

33. Minokoshi Y, Alquier T, Furukawa N, et al: AMP-kinase regulates food intake by responding to hormonal and nutrient signals in the hypothalamus. Nature 428: 569-574, 2004. 